\title{
Comparison of creep crack initiation and growth in four steels tested in HIDA
}

\author{
M. Tan, N. J. C. Celard, K. M. Nikbin, G. A. Webster \\ Department of Mechanical Engineering \\ Imperial College \\ London SW7 2BX
}

\begin{abstract}
In power generation plants and the chemical industries there is a need to assess the significance of defects which may exist in high temperature equipment operating in the creep range. For the life prediction methodology for cracked components developed under the HIDA (High Temperature Defect Assessment) Brite/Euram project, it is necessary to have a verifiable data-base of crack initiation and growth data in order to obtain relevant material properties for use in calculations. This paper examines the methods of analysis used. Four types of steels were tested in the programme. These were P22, 1CMV, 316LN and P91 in the parent, as welded and overaged conditions. The data have been obtained from seven participating laboratories. All the results were analysed in the same way using a programme called 'ZRATE' developed to follow the ASTM E1457-98 testing standard. The results are compared with other crack initiation and creep crack growth data in the literature and with predictions produced from creep data using a model of the cracking process. It has been found that in all cases the scatter in the data is less than for the generic data in the literature. It has also been found that creep crack initiation and growth data can be correlated most satisfactorily in terms of the creep fracture mechanics parameter $C^{*}$.
\end{abstract}

Keywords: Fracture mechanics, creep, crack initiation, crack growth

\section{Introduction}

Initially a survey was made of creep crack growth data at elevated temperatures on high strength steels [1]. It was found that there was significant batch to batch scatter in the data. In addition very little information was available in the literature on crack initiation from defects under creep conditions. The objective of the present experimental program was to produce creep crack initiation and growth properties for P22, 1CMV, 316LN and P91 steels in the parent, as welded and overaged conditions in order to develop a unified European life assessment methodology. 
Figure 1 and Table 1 report details of the material composition, conditions test temperatures, used for the steels that were examined. The creep crack growth tests were performed by all the partners (included in the Acknowledgement). The data were analysed using the software programme 'ZRATE' which applies the methodology proposed in ASTM E1457-98 [2]. This method of analysis of the data was used in order to unify the treatment of the output. Compact Tension (CT) of sizes $\mathrm{W}=50$ and $25 \mathrm{~mm}$, Single Edge Notch Tension (SENT) and Center Cracked Tension (CCT) specimens were used in the testing. Comparisons of crack initiation and growth were then made with respect to material condition, specimen geometry and size.

\section{Creep Crack Growth analysis}

The parameters used to describe creep crack growth rate $\dot{a}$ are the stress intensity factor $K$, the reference stress $\sigma_{r e f}$, and the creep fracture mechanics term $C^{*}$ [3-5]. They are used to describe and correlate data obtained for a range of materials and creep ductilities [2]. They are usually based on the following empirical laws:

$$
\begin{gathered}
\dot{a}=A \mathrm{~K}^{m} \\
\dot{a}=D C^{* \phi} \\
\dot{a}=H \sigma_{r e f}^{p}
\end{gathered}
$$

where $\mathrm{A}, \mathrm{D}, \mathrm{H}, \mathrm{m}, \varphi$ and $\mathrm{p}$ are material constants depending on temperature, and stress state.

Usually for brittle conditions eqn. (1) is most applicable, whereas eqn. (2) is relevant for most engineering situations and eqn. (3) to when failure is essentially by net section rupture. Although correlations with the stress intensity factor $K$ and the reference stress $\sigma_{\text {ref, }}$ were considered in this investigation only comparisons with $C^{*}$ are presented since it was found to be more appropriate [4,6-7]. The $C^{*}$ parameter was obtained from the general formula [4] used for experimental laboratory determinations,

$$
C^{*}=\frac{P \dot{\Delta}}{B_{n} W} F
$$

for a specimen of thickness $B_{n}$ between side-grooves, width $W$ and subjected to a load $P$. In this expression $\dot{\Delta}$ is the experimentally measured load point creep displacement rate and $F$ is 
a non-dimensional factor which depends on the stress sensitivity $n$ for multiaxial creep deformation, crack length $a$ and specimen geometry.

It has been found previously [3-7] for a wide range of materials that creep crack growth rate is most sensitive to creep failure strain and that it can be represented approximately by

$$
\dot{a}=\frac{3 C^{* 0.85}}{\varepsilon_{f}^{*}}
$$

where $\dot{a}$ is in $\mathrm{mm} / \mathrm{h}, \varepsilon_{f}^{*}$ is the appropriate failure strain as a fraction and $C^{*}$ is in $M J / m^{2} h$. In eqn. (5) $\varepsilon_{f}^{*}$ is the level of creep strain appropriate to the state of stress at the crack tip. For plane stress conditions $\varepsilon_{f}^{*}$ can be taken as the uniaxial creep ductility $\varepsilon_{f}$ and for plane strain conditions as $\varepsilon_{f} / 30$. This range describes the effects of constraint on crack growth due to both material properties and size/geometric factors. These bounds for plane stress/strain are also shown in some of the figures, which will be described below. Equations (2) and (5) are relevant to when steady state creep and creep damage conditions have been achieved at a crack tip. It should be noted that $\varepsilon_{f} / 30$ has been reduced from $\varepsilon_{f} / 50$ which was originally developed [6] from averaging the results obtained from three different void growth models [6] to cover a much wider range of materials and hence more conservative.

\section{Analysis for crack initiation}

There are available databases, which hold information relevant material data for specific models. However extensive databases do not exist for creep crack initiation chiefly because the information is not normally required for modelling and life assessment purposes and also because there is no unique criterion set to identify the initiation period in a cracked component. After comparison of crack extension data ranging from $0.05 \mathrm{~mm}$ to $1 \mathrm{~mm}$, the potential drop data and NDT results from components it was concluded that a crack growth increment of $d a=0.5 \mathrm{~mm}$ was an acceptable and a practical engineering criterion for defining crack initiation. This size was chosen after comparison of times to different crack extensions. In addition most partners reported that the accuracy of their PD measurements was in the order of $\pm 0.25 \mathrm{~mm}$.

Currently there are four models proposed in HIDA for estimating crack initiation. These are based on the so called stress at a distance $\sigma_{\mathrm{D}}[8]$, the crack opening displacement $\delta / C^{*}[9]$, 
two criteria [10] and the transient [11] approaches. Only the transient method is considered here.

\section{Transient model}

The initial stage of cracking exhibits a transient phenomenon due to the build up of damage at a crack tip prior to the onset of steady state behaviour. This can lead to an incubation period (as shown in Fig. 2) before measurable crack growth can be detected [11]. If the minimum crack extension that can be resolved reliably is $d a$ then the incubation period $t_{\mathrm{i}}$ is given by

$$
t_{\mathrm{i}}=\int_{0}^{\Delta a} \frac{d r}{\dot{a}}
$$

where $d r$ is distance from the crack tip. Bounds on $t_{i}$ can be obtained by representing cracking rate by its initial value $\dot{a}_{o}$ and its steady state value $\dot{a}$. The initial cracking rate can be approximated to the steady state rate in eqn. (2) by

$$
\dot{a}_{0}=\dot{a} /(n+1)
$$

The value of $n$ for most engineering materials is usually in the range 5-10, which suggests that $\dot{a}_{o}$ is approximately an order of magnitude less than its steady state value. Thus eqn. (2) for steady state conditions gives a lower bound incubation period of

$$
t_{\mathrm{iL}}=d a / D C^{* \phi}
$$

or using the approximate eqn. (5)

$$
t_{i L}=\frac{d a \varepsilon_{f}^{*}}{3 C^{* 0.85}}
$$

Alternatively if the incubation period is calculated from the initial transient cracking rate $\dot{a}_{o}$ determined from eqn. (7) the approximate upper-bound $t_{i U}$ to the initiation time becomes

$$
t_{i U}=\frac{(n+1) d a \varepsilon_{f}^{*}}{3 C^{* 0.85}}
$$

In eqn. (8-10) the incubation period is proportional to $\Delta a$. The limit of reliable crack detection is at best $\pm 100 \mu \mathrm{m}$ (which is the level set for standard CT testing in ASTM E1457-98) and can sometimes be as large as $0.5 \mathrm{~mm}$. This range is examined in the investigation. 


\section{Analysis of the data}

Constant load tests on standard compact tension geometries containing side-grooves were carried out on 4 types of steels (P22, 316-LN, 1CRMOV and P91) by partners [see acknowledgements]. The material condition and the test temperatures examined are shown in fig. 1 and Table 1. The ZRATE programme was used to analyse results and to identify the influence of creep ductility, specimen size, side-grooving and geometry on material crack initiation and growth properties. Figures 3-16 show the results. Predictions are made using eqns. (5), (9) and (10) and the average creep ductilities reported for each material assuming plane stress and strain conditions.

\section{P22 Material}

The P22 material has been extensively tested at ENEL for various loading conditions. The information derived from the tests is related to the base as well as the service-exposed materials. The results are shown in figs. 3-4. Fig. 3 shows cracking rate correlated with $C^{*}$ and fig. 4 shows the initiation times for each test versus $C^{*}$. The criterion for initiation was taken to be the time taken to extend the crack by $d a=0.5 \mathrm{~mm}$ and $C^{*}$ was calculated at this crack length. P22 was successfully correlated by the $C^{*}$ parameter and there appears to be no size or geometry effects within the narrow range of scatter and no effect is shown due to the HAZ material. The initiation times in fig. 4 show a large extent of scatter and are lower than the lowerbound predictions for the transient model. Fig. 3, for the crack growth, also shows the scatterband of data from the literature [1] for a temperature range of $538-570{ }^{\circ} \mathrm{C}$. In comparison there is reduced scatter in the batch specific HIDA tests compared to the data band from the literature.

Figs. 5 and 6 show the crack growth and initiation correlations for the P22 weld material with and without cavities and the ex-service P22 material without cavities (tested at SIMR). For the range tested there seems little effect due to the different conditions. Figs. 5 and 6 also shows the best fit line for the parent P22 (in figs. 3 and 4) for crack growth and initiation respectively. It is clear that the base material exhibits a longer initiation and show crack growth rate compared to the weld and HAZ materials. The trends suggest that initiation times for the parent P22 are considerably longer than for the ex-service material. The upper and lower bounds from eqns. (9) and (10) are plotted for initial crack extension of $0.5 \mathrm{~mm}$ in fig. 6. From the uniaxial tests it was observed that the maximum failure ductility for the parent 
P22 was about 0.45 whereas for the ex-service material the value was approximately 0.25 . The predictions for the bounds using the NSW model in equation 5 are also shown in figs. 3 and 5. The data is well bounded by the plane/stress/strain ranges for both material conditions.

\section{6-LN Stainless Steel Material}

The 316LN stainless steel, provided in plate form by CEA, was tested at $650{ }^{\circ} \mathrm{C}$. The graphs of crack initiation and growth rate against $C^{*}$ are shown in Figs. 7-8. As with the other materials, steady state crack growth was successfully correlated using the $C^{*}$ term. Fig. 7 also shows the scatter band of data from the literature [1] for different stainless steels at similar test temperatures. The current results lie in the mid region of the data bands. The NSW [6,7] prediction lines show that the failure is close to the plane stress region of cracking.

Crack growth initiation times for $d a=0.5$ are shown in fig 8 . Comparisons with the predictions based on eqns. (9) and (10) clearly indicate that the experimental data fall on the lowerbound at short test times and above the lowerbound predictions at longer test times. However given the scatter in the initiation data no final conclusion can be reached

\section{CrMoV Material}

Both cast and forged $1 \mathrm{CrMoV}$ materials were tested as CT specimens at SPG and MPA. The results produced are shown in figs. 9-12. The crack growth rates for the cast and the forged $1 \mathrm{CMV}$, at $530{ }^{\circ} \mathrm{C}$ are shown in figs. 9 and 11 . Some size and geometry effects are apparent in the forged steel (fig. 11). Figs. 9 and 11 also show the scatter bands of data from ref. [1] collected for $1 \mathrm{CrMoV}$ in cast and forged conditions data from outside HIDA for a temperature range of $538-600{ }^{\circ} \mathrm{C}$.

The NSW model [6,7] from eqn. (5) is also shown in figs. 9 and 11. The failure ductilities used for the cast material was 0.2 and for the forged 0.4 . It is clear that the cast material cracks faster than the forged material as observed from the respective best-fit lines shown in figs. 9 and 11. It is likely that the lower failure strain in the cast material increases constraint at the crack tip and reduces failure life.

Initiation times versus $C^{*}$ are also shown for the cast and forged 1CMV steel in figs. 10 and12 respectively. It is shown that the results lie close to the upper bound of the predictions using 
eqns. 10 and 12. It is also clear that initiation times are longer for the forged material than the cast when the best fit line for each are compared.

\section{P91 Material}

The P91 material has been extensively tested at SPG for various loading conditions, and information taken from the tests for two thicknesses of CT and one size of SENT, is related to the base as well as to the HAZ and the service exposed materials conditions. The results are shown in figs. 13 and 14. In fig. 13 although the data fall in a scatter band virtually bounded by the NSW model (eqn. (5) ) using a failure strain of 0.4 there is a tendency for the HAZ material to crack at a faster rate at the same $C^{*}$ than the base (parent) material. The range of slopes for $\phi$ in eqn. (5) were obtained and their values range between 0.65-0.95. The effects due to specimen size and geometry are all within the scatterband with no observable trends. Fig. 13 also shows the scatter band of data from ref. [1] (Collected from P91 base and weld tests in the temperature range of $538-593{ }^{\circ} \mathrm{C}$ ). The crack growth rates in this case are shown to be slightly higher than for the HIDA materials.

Fig. 14 show the initiation data with respect to $C^{*}$. By considering the initial crack extension of to $d a=0.5 \mathrm{~mm}$ it is seen from fig. 14 that the data is bounded by the transient initiation model (eqns. $(9,10))$ although at shorter incubation times the data correlates better with the lowerbound and for longer times (i.e. at lower loads) the data falls on the upperbound.

\section{Conclusions}

Tests have been performed by the HIDA group to investigate the creep crack initiation and growth characteristics of five types of steels for use in the unified European life assessment methodology proposed in HIDA. Creep crack growth data from the HIDA tests have been analysed and compared with data bands extracted from the literature [1]. It has been found that the scatter, although improved when compared to data from the literature [1], exists even in a controlled Round Robin testing programme using the same batches of material, agreed testing methods and a unified analysis procedure. The aim of this work was to produce a unified analysis of the data, present an overall comparison of the crack growth data and produce relevant material crack initiation and growth data that can be used in the HIDA Knowledge Based System (KBS). This has been achieved to a good degree. However it is clear from the findings that the inherent scatter in results may need to be dealt with using a improved statistical or probabilistic methods. 


\section{Acknowledgements}

The authors would like to thank the European Commission for their financial contribution and

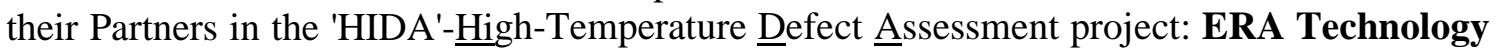
(UK); Imperial College (UK); MPA Stuttgart (Germany); Siempelkamp (Germany); CEA (France); EDF (France); SAQ Kontroll/SIMR (Sweden); ENEL (Italy); Petrogal/ISQ (Portugal); Metsearch (Netherlands); Framatome (France) for the provision of data.

\section{References}

1. Shibli, I. A., Al Abed, B. and Nikbin, K.' Scatter bands in creep and fatigue crack growth rates in high temperature plant materials data' Journal of Materials at High Temperature, Vol. 15, Int. HIDA Conf., 1998.

2. ASTM E 1457-98, 1998, "Standard Test Method for Measurement of Creep Crack Growth Rates in Metals", ASTM Standards 03.01, pp. 1031-1043.

3. Webster, G. A., Nikbin, K., Chorlton, M. R., Cellard, N.J.C., Ober, M., 'A comparison of High temperature defect assessment methods', Journal of Materials at High Temperature, Vol. 15, Int. HIDA Conf., 1998.

4. Webster, G. A., And Ainsworth, R. A., "high temperature component life assessment", Chapman \& Hall, 1994.

5. British Standards- $7910: 1999$, Guide to methods of assessing the acceptability of flaws in fusion welded structures, BSI, London, 1999.

6. Nikbin, K. M., Smith, D. J., and Webster, G. A., 1986, "Prediction of Creep Crack Growth from Uni-Axial Creep Data”, Proc. Roy. Soc., Series A, 396, pp.183-193.

7. Nikbin, K. M., Smith, D. J., and Webster, G. A., 1986 “An Engineering Approach to the Prediction of Creep Crack Growth”, J. Eng. Mat. and Tech., ASME, 108, pp.186-191.

8. AFCEN (1985) 'Design and construction rules for mechanical components of FBR nuclear islands' RCC-MR, Appendix A16, AFCEN, Paris.

9. Ainsworth, R.A., Chell, G.G., Coleman, M.C., Goodall, I.W., Gooch, D.J., Haigh, J.R., Kimmins, S.T. and Neate, G.J., 1987, “CEGB Assessment Procedure for Defects in Plant Operating in the Creep Range", Fat. and Fract. of Eng. Mat and Struct., 10, pp. 115-127.

10. Ewald, J. \& Keienburg, K-H, 'A Two Criteria Diagram For Creep Crack Initiation', Int. Conf. Creep, Tokyo, April 1986, 173-178.

11. Kaguchi, H., Djavanroodi, F., Nikbin, K. M., Webster, G. A., 'Prediction of transient effects during the early stages of creep crack growth', Ed. K.T. Rie, published in the proceedings of the DVM Conference, Berlin, Sept. (1994). 
Figure 1: Categories of material conditions tested in HIDA

a)

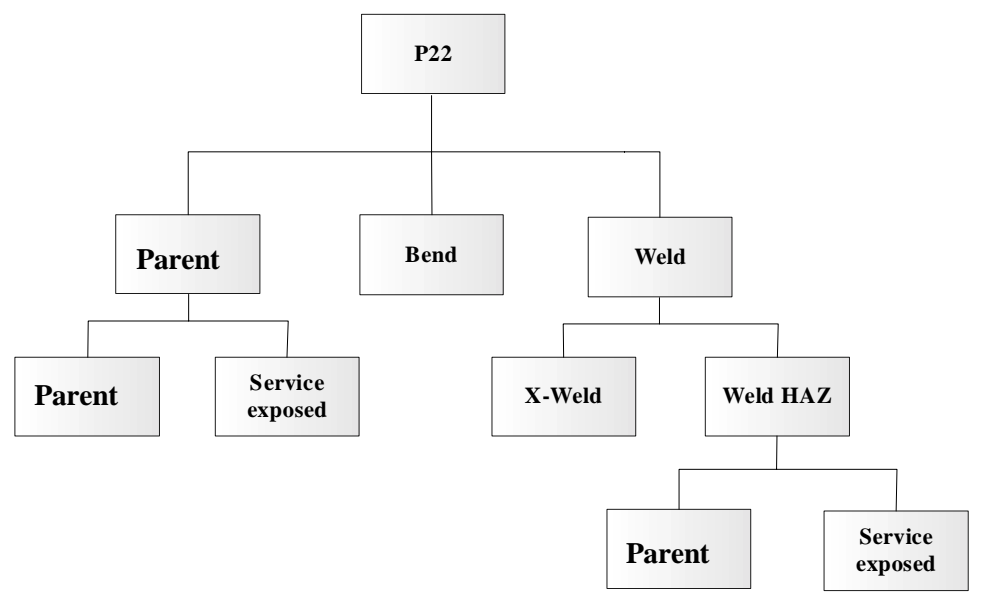

Figure 1(a): P22 - material tested at $565{ }^{\circ} \mathrm{C}$

b)

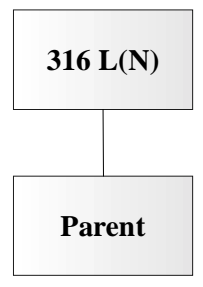

c)

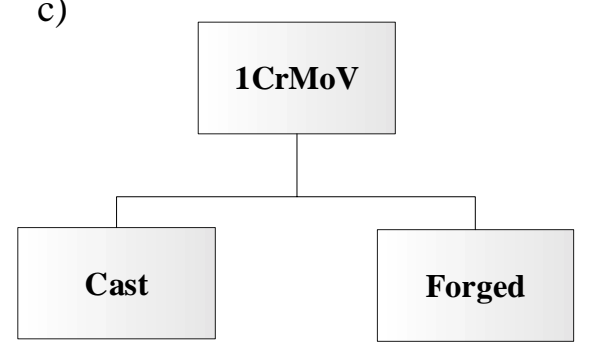

Figure 1: (b): $316 \mathrm{~L}(\mathrm{~N})$ - material tested at $650{ }^{\circ} \mathrm{C}$ and 1 (c): $1 \mathrm{CrMoV}$ - material tested at $530{ }^{\circ} \mathrm{C}$

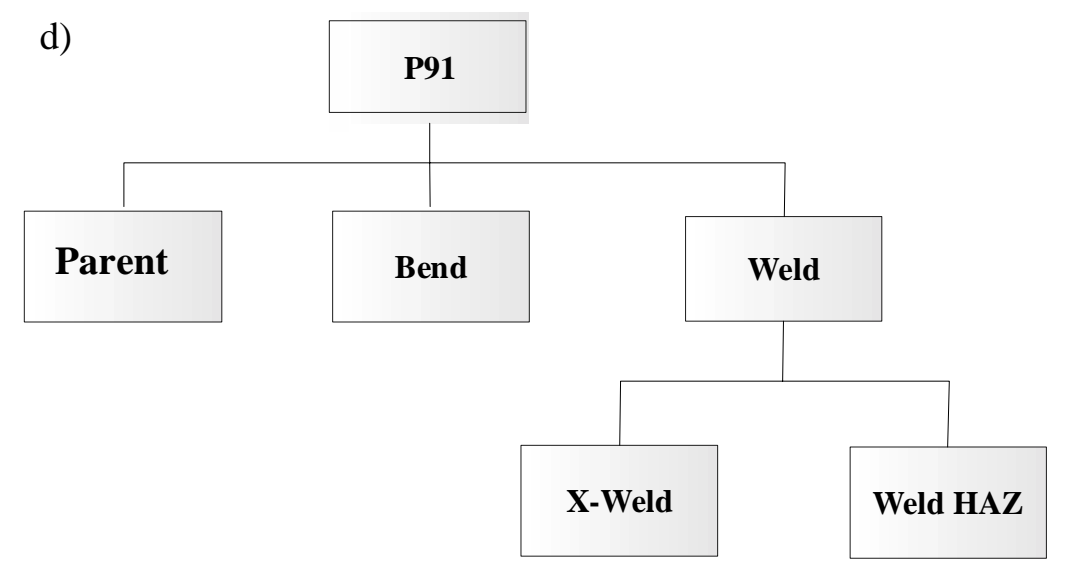

Figure 1(d): P91 - material tested at $625^{\circ} \mathrm{C}$ 
Table 1: Chemical composition of the parent 'HIDA' materials

Table 1(a): Material - P22

\begin{tabular}{|c|c|c|c|c|c|c|c|c|c|c|c|}
\hline & $\mathrm{C}$ & $\mathrm{Mn}$ & $\mathrm{Si}$ & $\mathrm{S}$ & $\mathrm{P}$ & $\mathrm{Cr}$ & $\mathrm{Ni}$ & $\mathrm{Mo}$ & $\mathrm{V}$ & $\mathrm{N}$ & $\mathrm{Al}$ \\
\hline P22 & 0.101 & 0.443 & 0.206 & 0.015 & 0.024 & 2.070 & 0.099 & 0.939 & 0.180 & 0.013 & $<0.01$ \\
\hline
\end{tabular}

Table 1(b): Material - $316 \mathrm{~L}(\mathrm{~N})$ batch SD

\begin{tabular}{|l|l|l|l|l|l|l|l|l|l|l|l|}
\hline $\mathrm{C}$ & $\mathrm{Mn}$ & $\mathrm{Si}$ & $\mathrm{S}$ & $\mathrm{P}$ & $\mathrm{Cr}$ & $\mathrm{Ni}$ & $\mathrm{Mo}$ & $\mathrm{Ti}$ & $\mathrm{Nb}$ & $\mathrm{N}$ & $\mathrm{Cu}$ \\
\hline 0.038 & 1.830 & 0.313 & 0.02 & 0.036 & 17.3 & $11 . .9$ & 2.46 & $<0.01$ & $<0.01$ & 0.067 & 0.27 \\
\hline
\end{tabular}

Table 1(c):Material - 1CrMoV

\begin{tabular}{|l|c|c|c|c|c|c|c|c|c|c|c|c|}
\hline & $\mathrm{C}$ & $\mathrm{Mn}$ & $\mathrm{Si}$ & $\mathrm{S}$ & $\mathrm{P}$ & $\mathrm{Cr}$ & $\mathrm{Ni}$ & $\mathrm{Mo}$ & $\mathrm{V}$ & $\mathrm{Ti}$ & $\mathrm{Cu}$ & $\mathrm{Al}$ \\
\hline Cast & 0.158 & 0.660 & 0.398 & 0.004 & 0.007 & 1.370 & 0.280 & 1.030 & 0.237 & 0.029 & 0.06 & $<0.005$ \\
\hline Forged & 0.290 & 0.390 & 0.318 & 0.002 & 0.007 & 1.760 & 0.300 & 1.130 & 0.294 & 0.014 & 0.07 & $<0.005$ \\
\hline
\end{tabular}

Table 1(d): $\quad$ Material - P91

\begin{tabular}{|c|c|c|c|c|c|c|c|c|c|c|c|c|}
\hline & $\mathrm{C}$ & $\mathrm{Mn}$ & $\mathrm{Si}$ & $\mathrm{S}$ & $\mathrm{P}$ & $\mathrm{Cr}$ & $\mathrm{Ni}$ & $\mathrm{Mo}$ & $\mathrm{V}$ & $\mathrm{Cu}$ & $\mathrm{N}$ & $\mathrm{Al}$ \\
\hline P91 & 0.09 & 0.409 & 0.369 & 0.013 & 0.028 & 8.440 & 0.270 & 0.920 & 0.240 & 0.040 & 0.038 & 0.070 \\
\hline
\end{tabular}

Table 2: Summarized creep crack growth properties from ref: [1]

\begin{tabular}{|cccccc|}
$\varepsilon_{f}=$ uniaxial creep ductility. $d a / d t=D C^{*} \phi$ & $C^{*}$ is in $M J / m^{2} h, \dot{a}$ in $\mathrm{mm} / \mathrm{h}$ \\
\hline Material & Condition & Temp ${ }^{\circ} C$ & $\varepsilon_{f}$ & $D$ & $\phi$ \\
P22 & parent & 565 & 0.37 & 5.2 & 0.85 \\
P22 & weld & 565 & 0.07 & 3.5 & 0.64 \\
P91 & parent & - & 0.13 & 1.8 & 0.63 \\
P91 & weld & - & 0.02 & 20 & 0.80 \\
1Cr_cast & parent & 530 & 0.22 & 4.2 & 0.72 \\
1Cr_forged & parent & $540-550$ & 0.17 & 2.5 & 0.69 \\
316SS & parent & 550.625 & - & 2.3 & 0.74 \\
316HAZ & HAZ & $510,525,560$ & - & 2.4 & 0.69
\end{tabular}

$\mathrm{D}$ is taken from the best average line through all the data irrespective of geometry

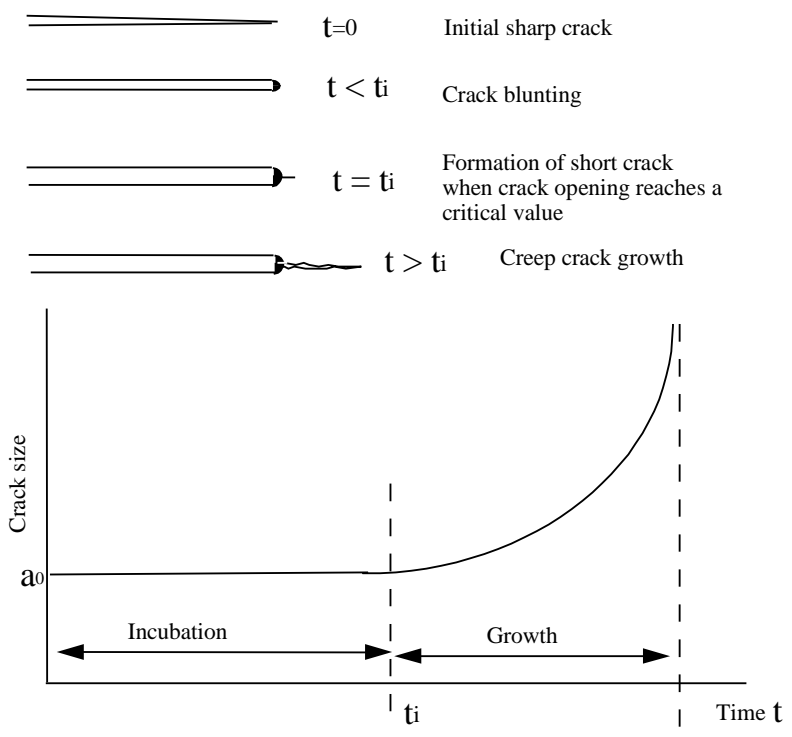

Figure 2: Schematic behaviour of a crack at elevated temperature 
$\mathrm{CR}=$ Circumferential parent material, $\mathrm{HAZ}=$ the heat affected zone material, $\mathrm{LN}=$ longitudinal parent material, $\mathrm{BEND}=$ is pre-bent material, $\mathrm{CT}=$ comp act tension, $\mathrm{SENT}=$ single edge notch tension, $\mathrm{W}=$ specimen width .

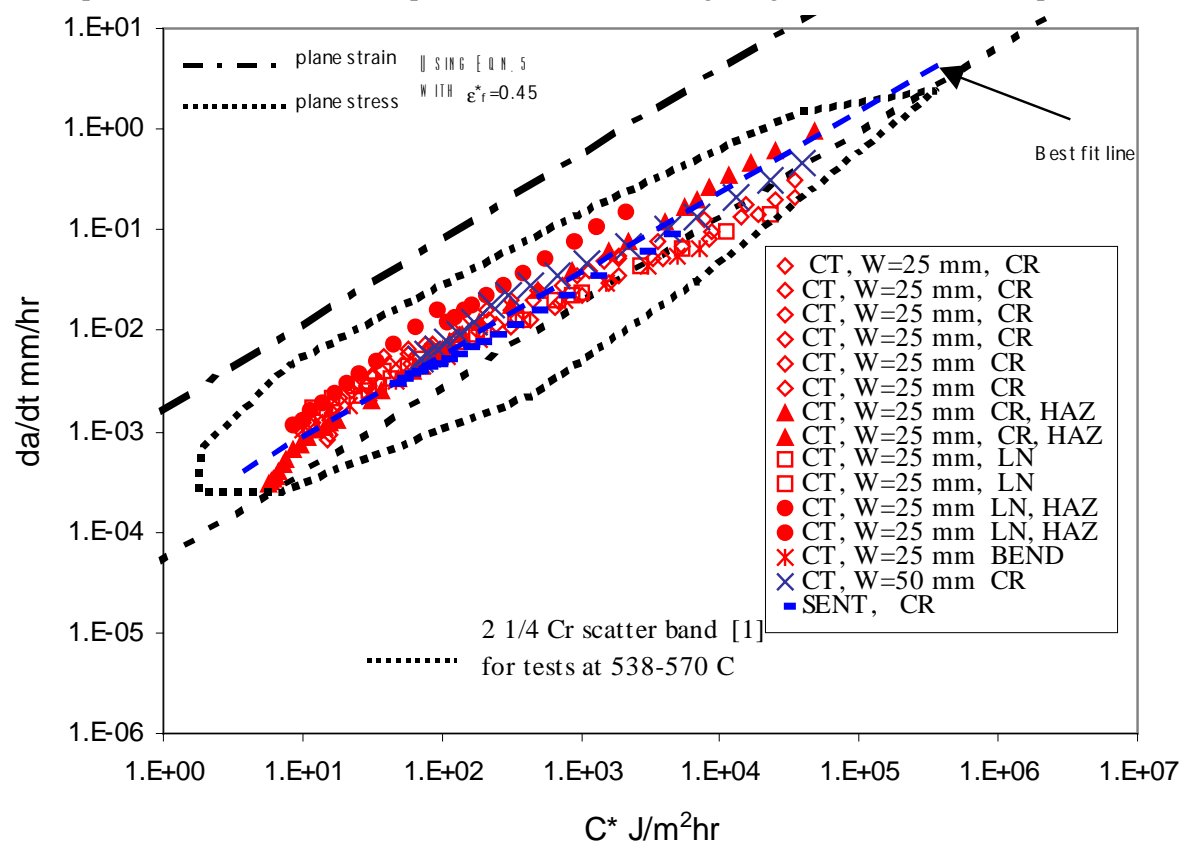

Figure 3: Graph of crack rate versus $C^{*}$ cycle for parent and HAZ P22 material tested at 565 ${ }^{\circ} \mathrm{C}$.. The scatterband compare the data from reference 1 on P22 for a test temperature range of $538-570{ }^{\circ} \mathrm{C}$. The plane stress/strain prediction lines are taken from eqn. (5). W=width

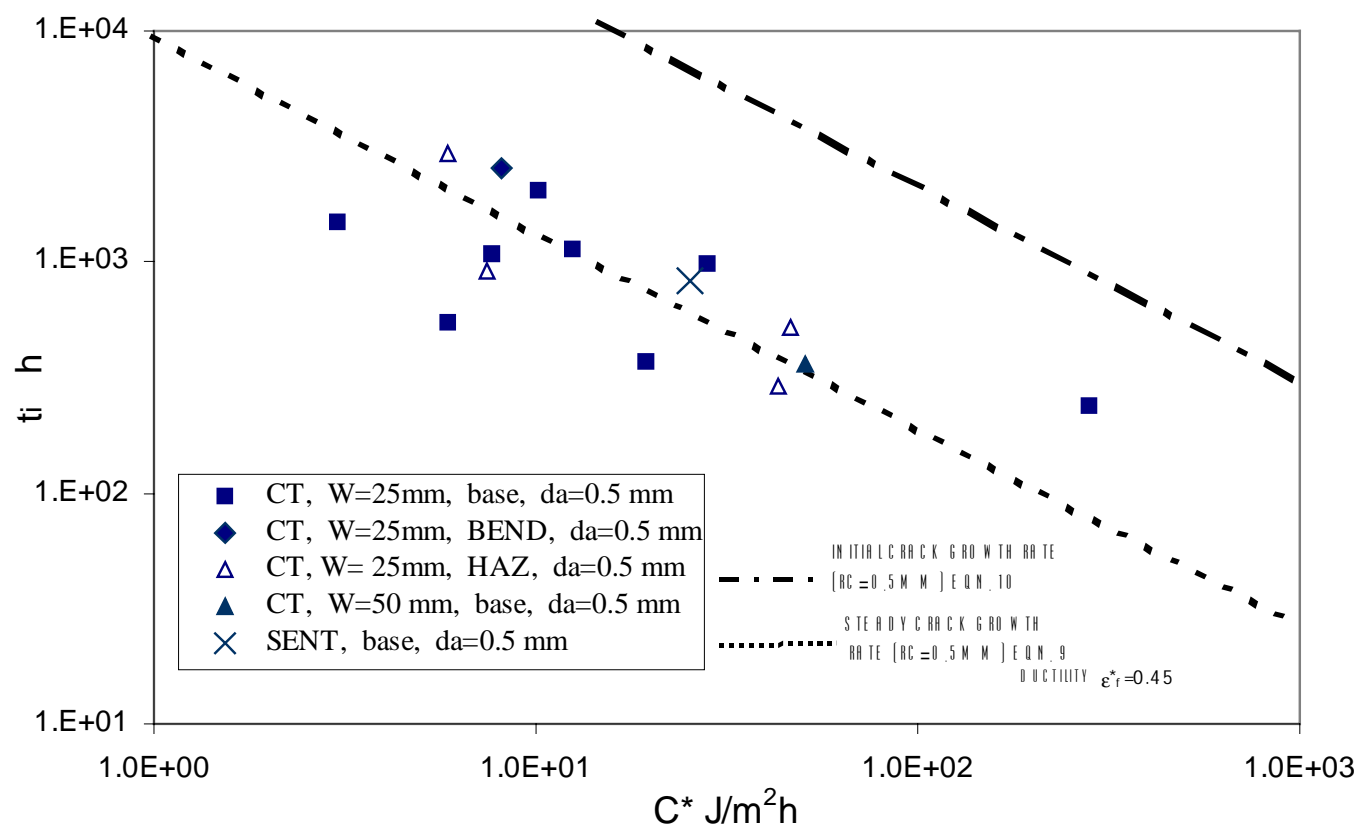

Figure 4: Graph of crack initiation time $t i \vee C^{*}$ for parent P22 material tested at $565{ }^{\circ} \mathrm{C}$ over the crack initiation length of $d a=0.5 \mathrm{~mm}$. The prediction from eqns. $(9,10)$ use $d a=0.5 \mathrm{~mm}$. 


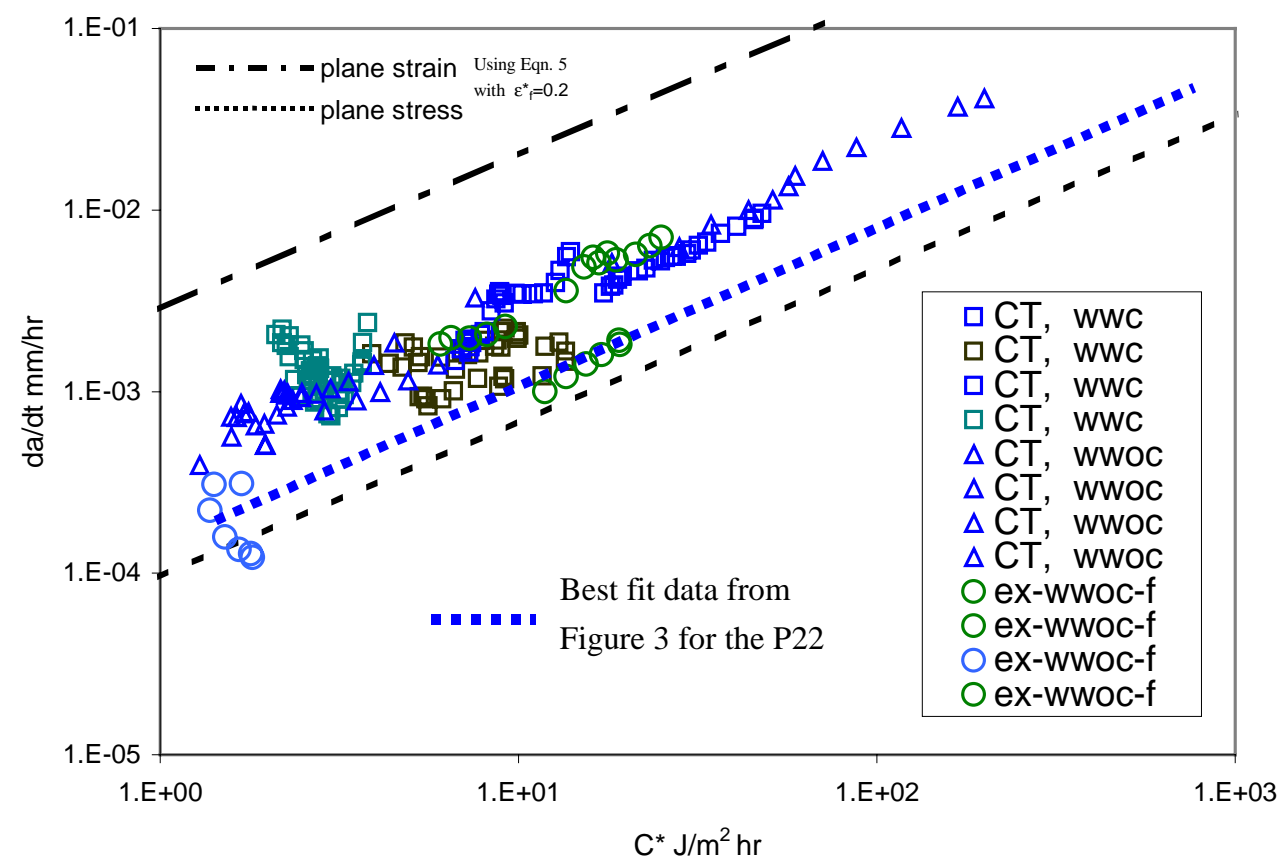

Figure 5: Graph of crack growth rate v $C^{*}$ for $\mathrm{P} 22$ weld material tested at $565^{\circ} \mathrm{C}$. The plane stress/strain prediction lines are taken from eqn. (5)

$w w c=$ weld with cavities, wwoc=weld without cavities and $($ ex-wwoc- $f($ ex-service HAZ $))$

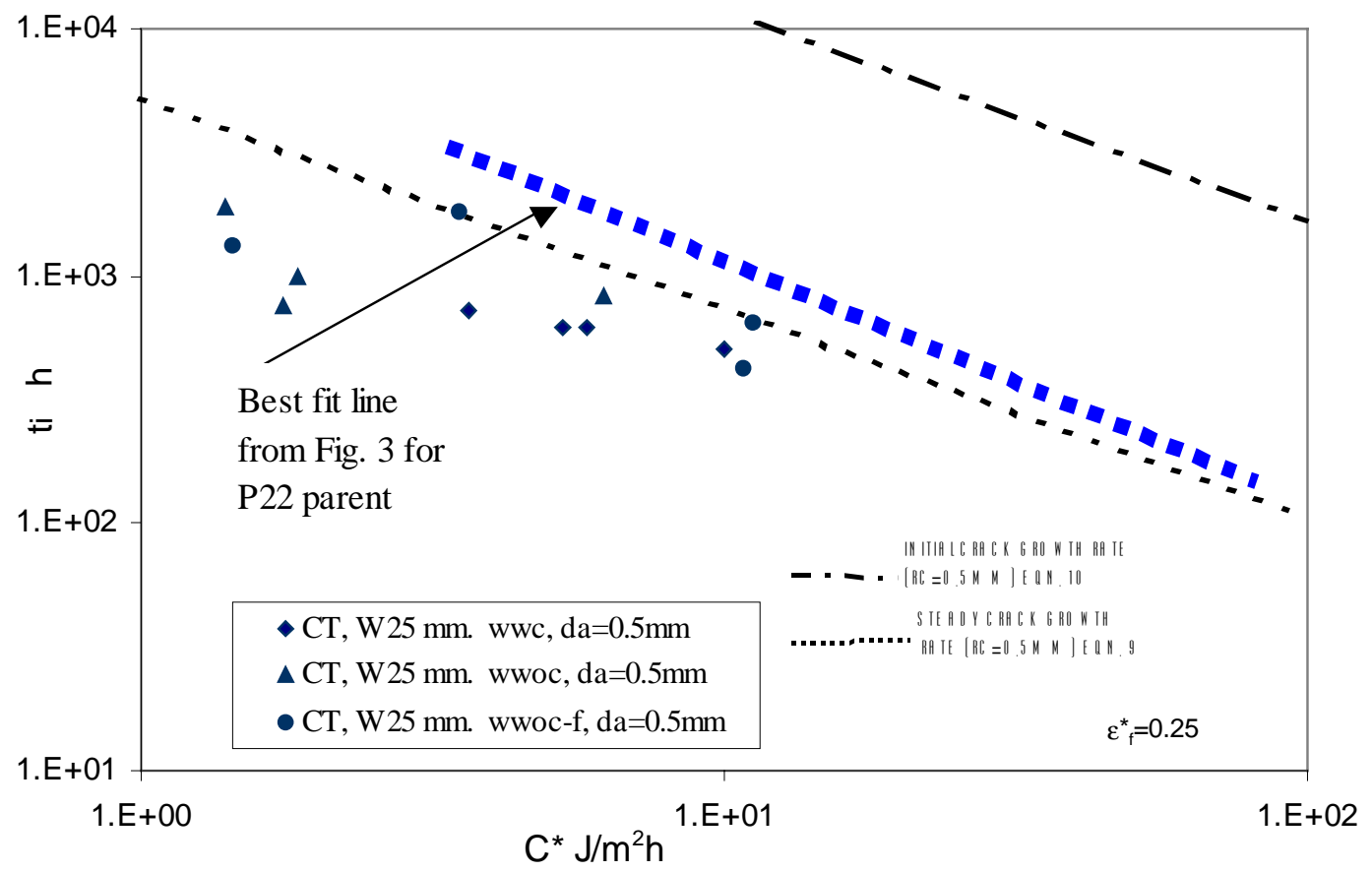

Figure 6: Graphs of crack initiation time $t_{i}$ v $C^{*}$ for ex-service P22 material tested at $565{ }^{\circ} \mathrm{C}$ over the crack length $d a=0.5 \mathrm{~mm}$. The prediction from eqns. $(9,10)$ use $d a=0.5 \mathrm{~mm}$. The data is compared to initiation times for the parent P22 shown in Fig. 4 


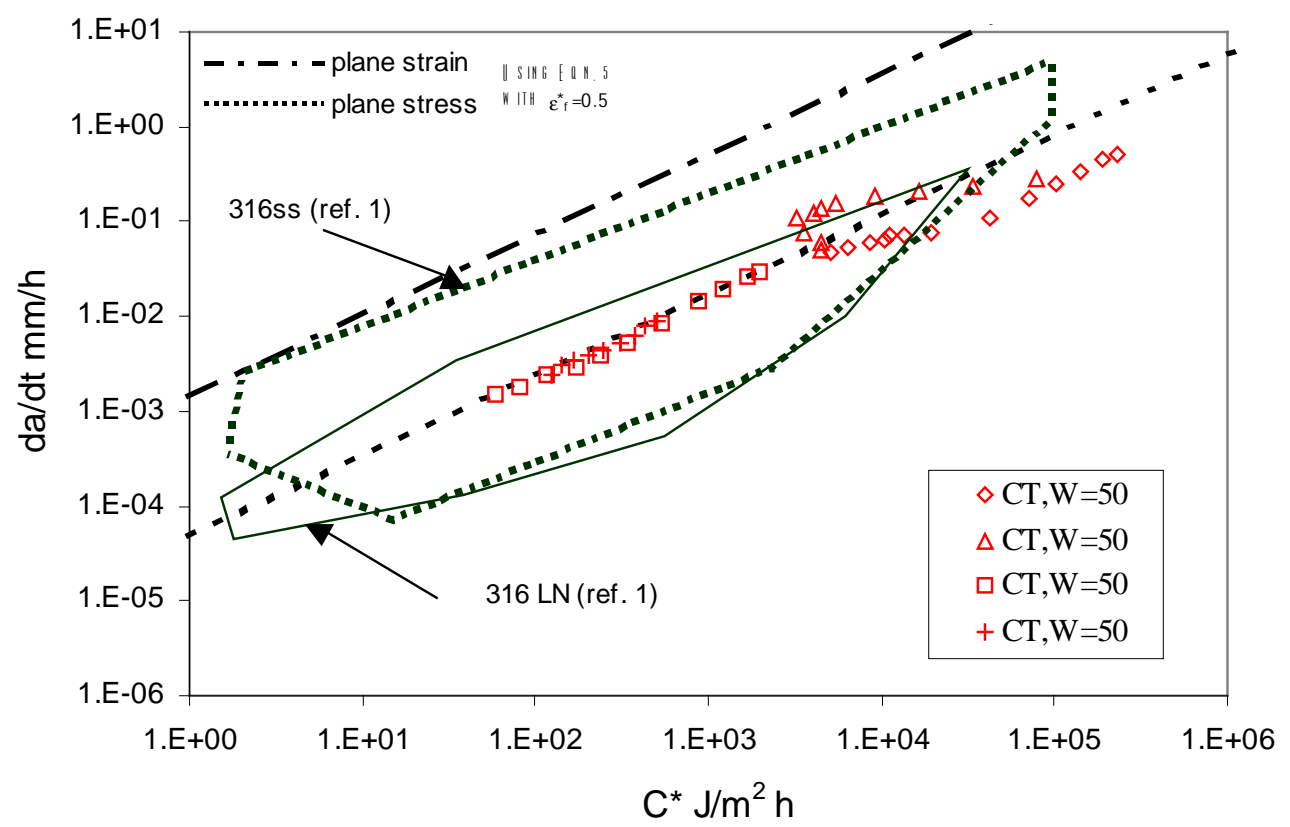

Figure 7: Graph of crack growth $\mathrm{v} C^{*}$ for $316 \mathrm{LN}$ material tested at $650{ }^{\circ} \mathrm{C}$. using CT and SENT specimens. The scatterbands shown are from various stainless steels data taken from ref. [1]. The plane stress/strain predictions are taken from eqn. (5) using $\varepsilon *_{\mathrm{f}}=0.5$

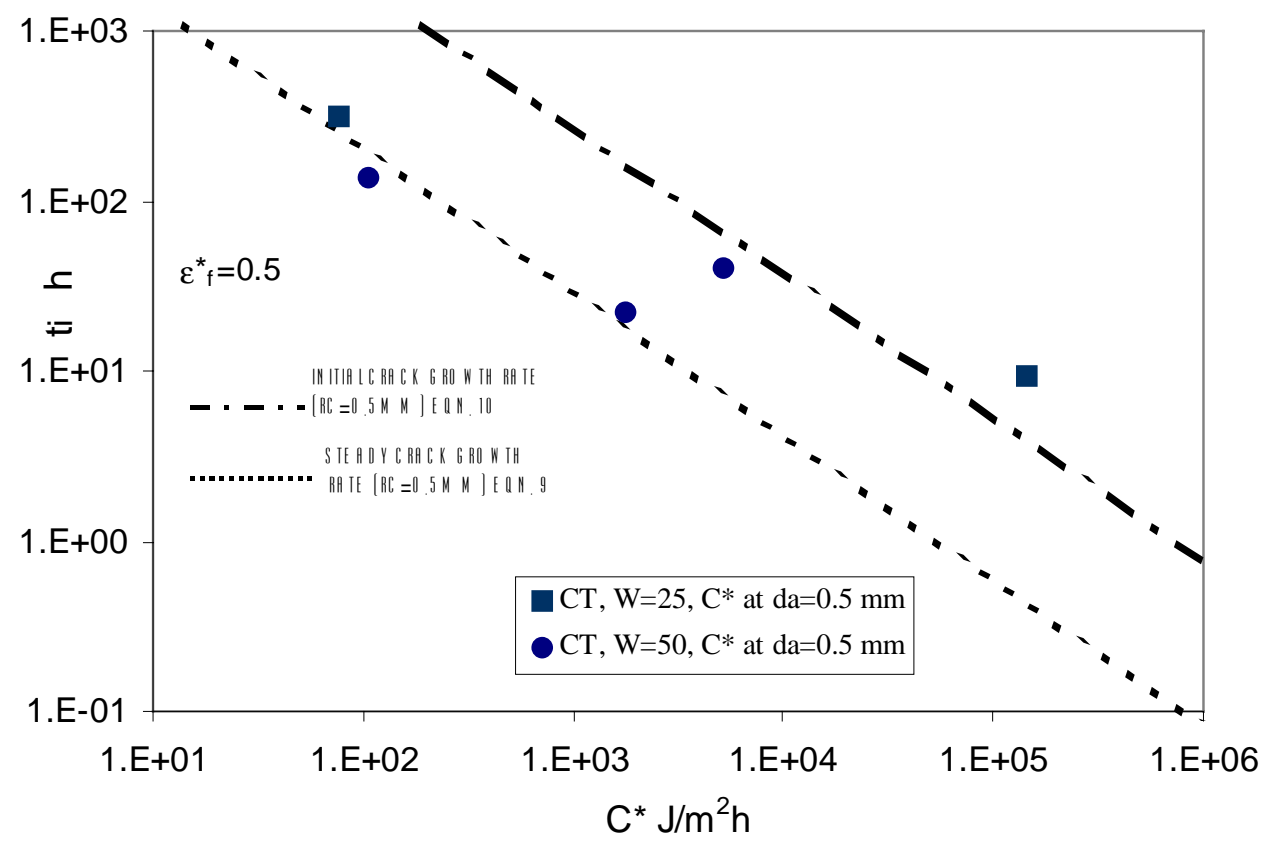

Figure 8: Graph of crack initiation time $t_{i} \vee C^{*}$ for $316 \mathrm{LN}$ material tested at $650{ }^{\circ} \mathrm{C}$ over the crack extension length of $0.5 \mathrm{~mm}$. The prediction from eqns. $(9,10)$ use $d a=0.5 \mathrm{~mm}$ 


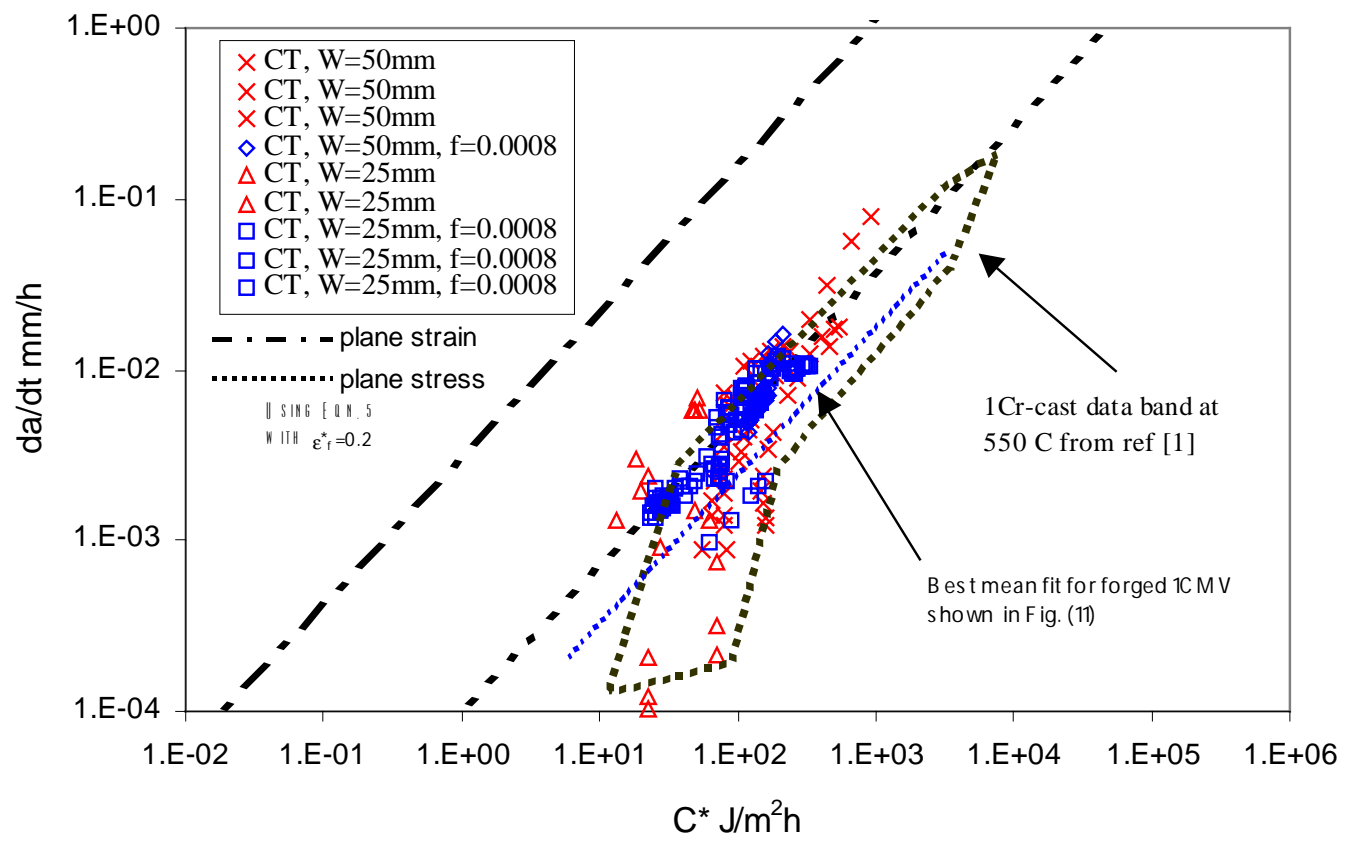

Figure 9: Graph of crack growth versus $C^{*}$ for Cast $1 \mathrm{CrMoV}$ steel tested at $530{ }^{\circ} \mathrm{C}$ showing 2 sizes of CT specimens and low frequency data. The scatterband is from $1 \mathrm{Cr}$ Cast data taken from ref. [1]. The best fit for the 1Cr Forged from Fig. 11 is also shown. The plane stress/strain prediction lines are taken from eqn. (5)

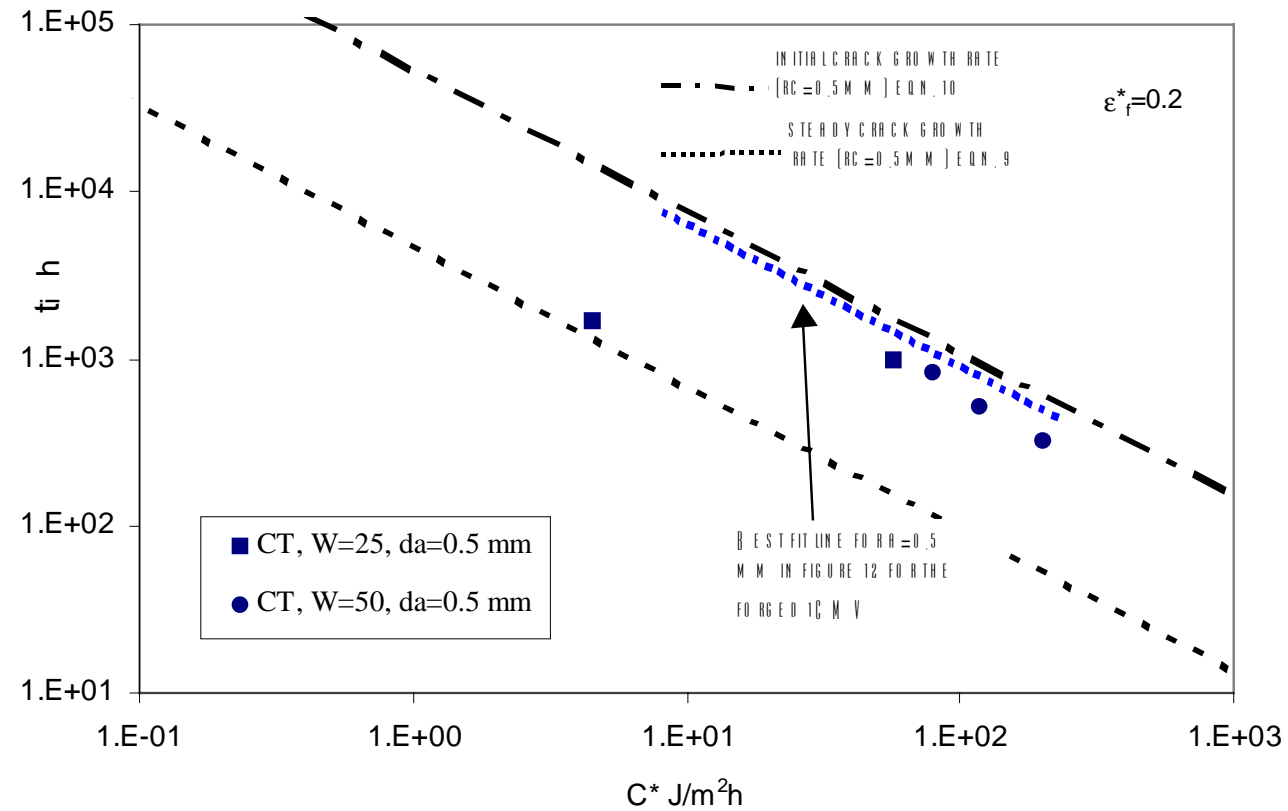

Figure 10: Graph of crack initiation time $t_{i}$ v $C^{*}$ for Cast $1 \mathrm{CrMoV}$ steel tested at $530{ }^{\circ} \mathrm{C}$. using 2 sizes of CT specimens over the crack extension length of $0.5 \mathrm{~mm}$. The prediction from eqns. $(9,10)$ use $d a=0.5 \mathrm{~mm}$. The best fit for the $1 \mathrm{Cr}$ Forged from Fig. 12 is also shown 


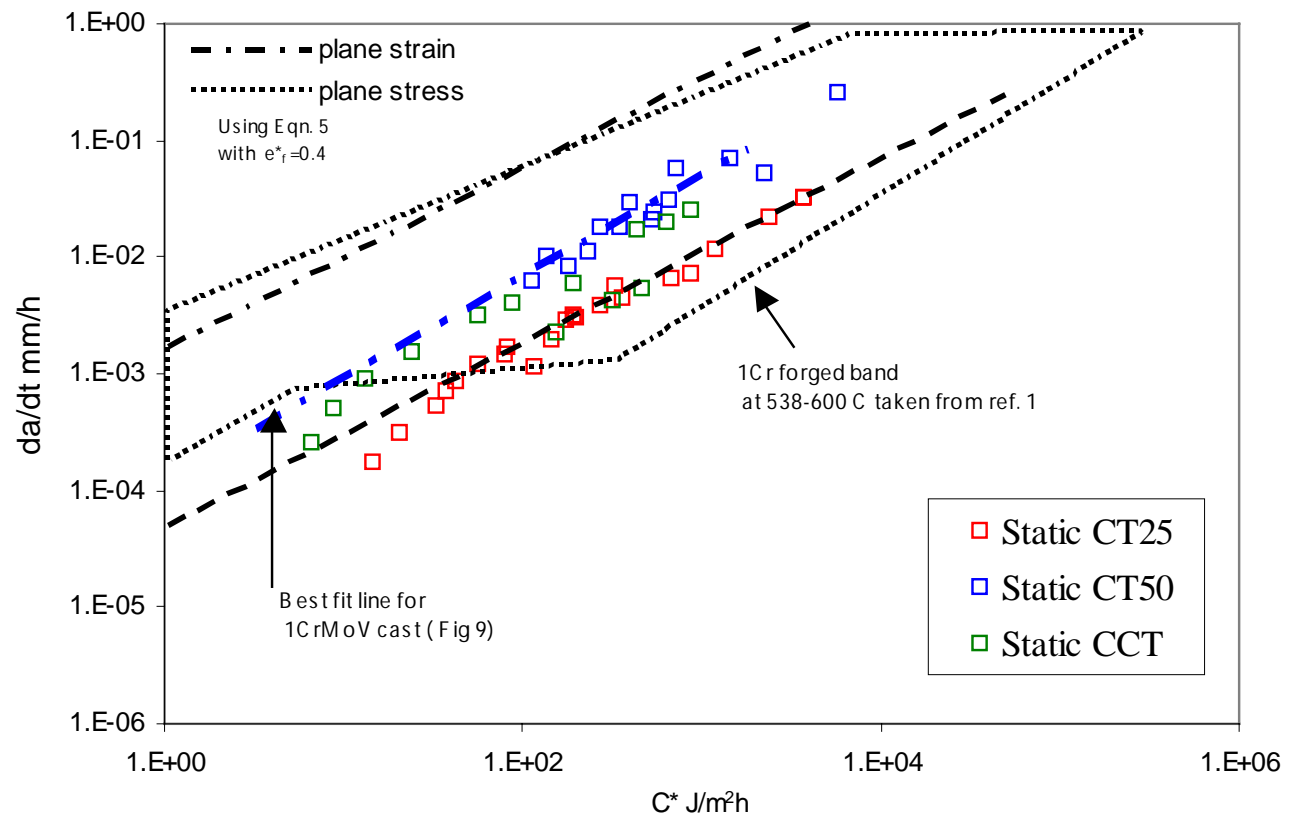

Figure 11: Graph of crack growth versus $C^{*}$ for forged $1 \mathrm{CrMoV}$ steel tested at $530{ }^{\circ} \mathrm{C}$ showing 2 sizes of $\mathrm{CT}$ and one size $\mathrm{CCT}$ specimen. The scatterband is from $1 \mathrm{Cr}$ forged data taken from reference [1]. Also compared is the best mean fit $1 \mathrm{Cr}$ cast data shown in figure 9 . The plane stress/strain prediction lines are taken from eqn. (5) using the forged material creep ductility

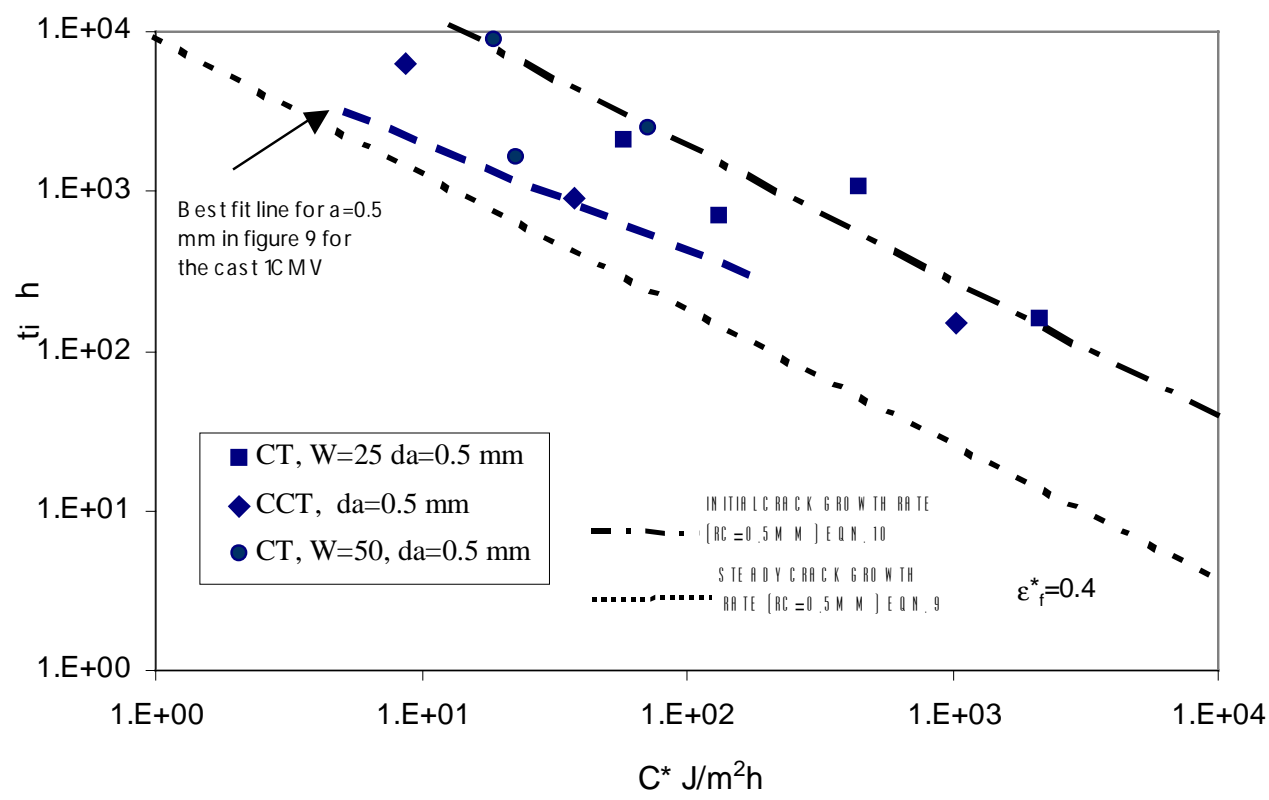

Figure 12: Graph of crack initiation time $t_{i} \vee C^{*}$ for forged $1 \mathrm{CrMoV}$ steel tested at $530{ }^{\circ} \mathrm{C}$, showing 2 sizes of CT and one size CCT specimen., over the crack extension of $0.5 \mathrm{~mm}$.

The prediction from eqns. $(9,10)$ use $d a=0.5 \mathrm{~mm}$ 


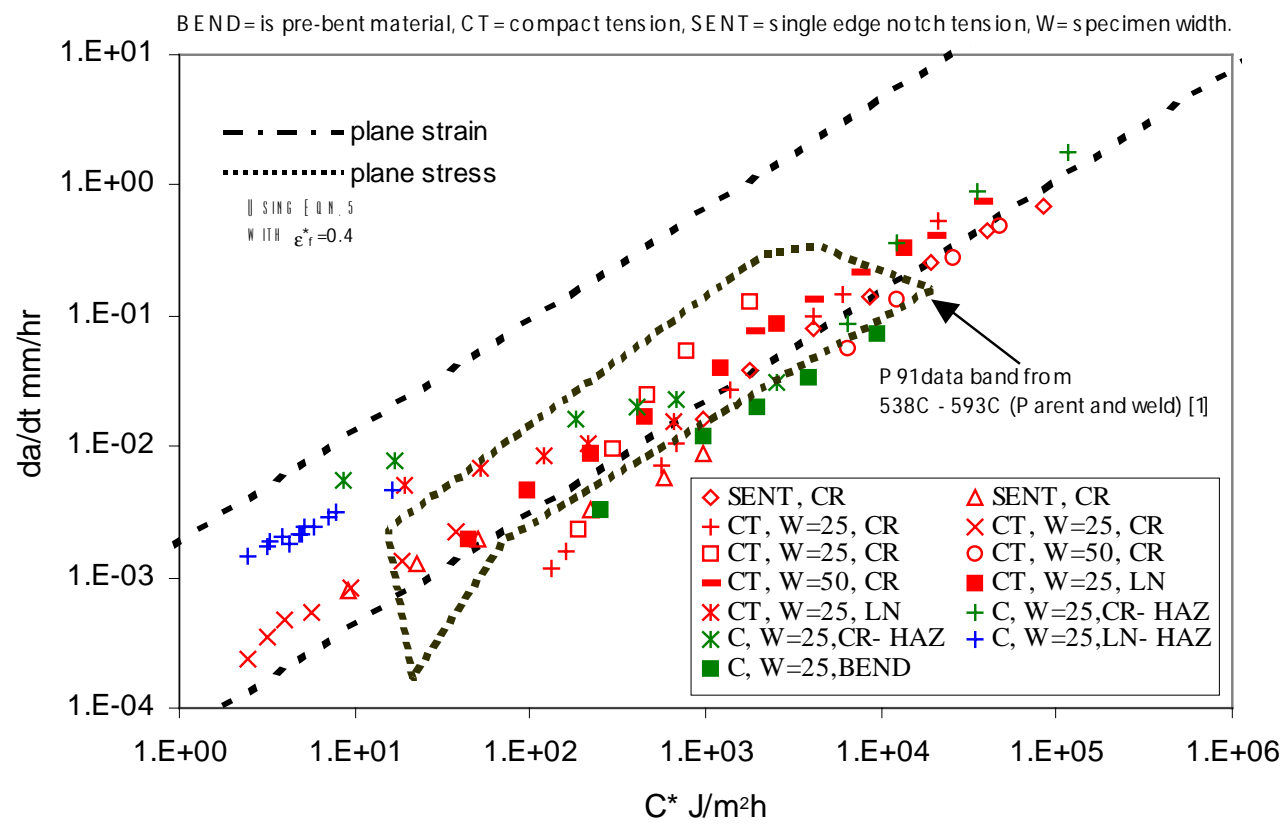

Figure 13: Graph of crack growth v $C^{*}$ for parent, and HAZ P91 material tested at $625{ }^{\circ} \mathrm{C}$ using CT and SENT geometries. The scatterband is from P91 (tested in the range of 538-593 ${ }^{0} \mathrm{C}$ ) data from ref. [1]. The plane stress/strain prediction lines are taken from eqn. (5)

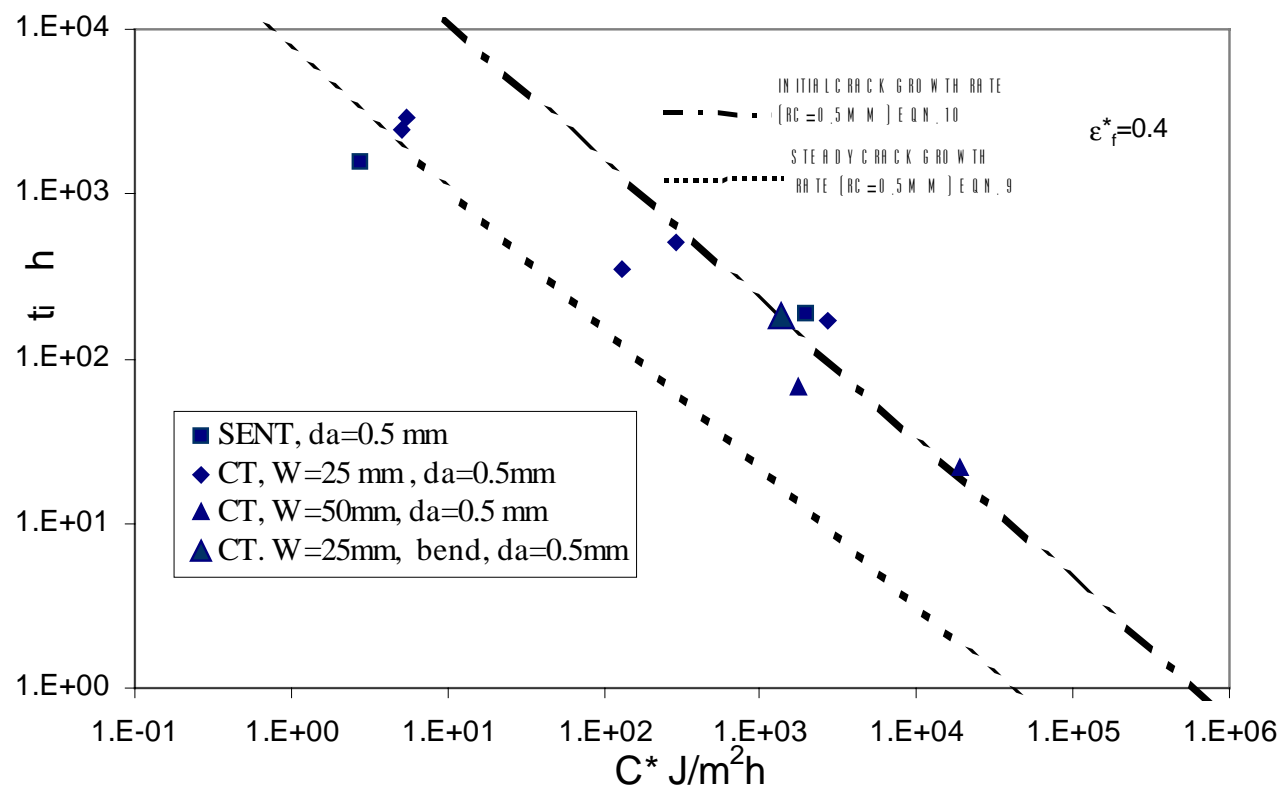

Figure 14: Graph of crack initiation time $\mathrm{v} t_{i} \quad \mathrm{v} C^{*}$ for parent P91 material tested at $625{ }^{\circ} \mathrm{C}$ using different geometries over a crack extension of $0.5 \mathrm{~mm}$. The prediction from eqns. $(9,10)$ use $d a=0.5 \mathrm{~mm}$ 